\title{
Numerical Study on the Boundary Value Problem by Using a Shooting Method
}

\author{
Md. Mizanur Rahman", ", Mst. Jesmin Ara ${ }^{2}$, Md. Nurul Islam¹, Md. Shajib Ali ${ }^{1}$ \\ ${ }^{1}$ Dept. of Mathematics, Faculty of Applied Science and Technology, Islamic University, Kushtia, Bangladesh \\ ${ }^{2}$ Department of Political Science, National University, Gazipur, Dhaka, Bangladesh
}

\section{Email address:}

mizan_iu@yahoo.com (M. M. Rahman), jesmin_nu@yahoo.com (M. J. Ara), nurul_math_iu@yahoo.com (M. N. Islam), shajib_301@yahoo.co.in (M. S. Ali)

\section{To cite this article:}

Md. Mizanur Rahman, Mst. Jesmin Ara, Md. Nurul Islam, Md. Shajib Ali. Numerical Study on the Boundary Value Problem by Using a Shooting Method. Pure and Applied Mathematics Journal. Vol. 4, No. 3, 2015, pp. 96-100. doi: 10.11648/j.pamj.20150403.16

\begin{abstract}
In the present paper, a shooting method for the numerical solution of nonlinear two-point boundary value problems is analyzed. Dirichlet, Neumann, and Sturm- Liouville boundary conditions are considered and numerical results are obtained. Numerical examples to illustrate the method are presented to verify the effectiveness of the proposed derivations. The solutions are obtained by the proposed method have been compared with the analytical solution available in the literature and the numerical simulation is guarantee the desired accuracy. Finally the results have been shown in graphically.
\end{abstract}

Keywords: Boundary Value Problem, Shooting Method, Numerical Simulation and MATLAB Programming

\section{Introduction}

There are many linear and nonlinear problems in science and engineering, namely second order differential equations with various types of boundary conditions, are solved either analytically or numerically. Two-point boundary value problems occur in a wide variety of problem such as modeling of chemical reactions, the boundary layer theory in fluid mechanics and heat power transmission. The wide applicability of boundary value problems in engineering and sciences calls for faster and accurate numerical methods. Many authors have attempted to obtain higher accuracy rapidly by using a numerous methods. The shooting method to compute eigen-values of fourth-order two-point boundary value problems studied by D. J. Jones [1].Wang et al [2] investigated application of the shooting method to second order multi point integral boundary value problems. Kwong and Wong [3] have studied the shooting method and non-homogeneous multipoint BVPs of second-order ODE. Abd-Elhameed et al [4] have investigated a new wavelet collection method for solving second-order multipoint boundary value problems using Chebyshev polynomials of the third and fourth kinds. See [5] studied nonlinear two point boundary value problem using two step direct method. Meade et al [6] discussed about the shooting technique for the solution two- point boundary value problems. Rahman et al [7] have studied numerical Solutions for Second Order Boundary Value Problems using Galerkin Method. Fatullayev et al [8] investigated numerical solution of a boundary value problem for a second order Fuzzy differential equation. Granas et al [9] investigated the shooting method for the numerical solution of a class of nonlinear boundary value problems. Cole and Adeboye [10] studied an alternative approach to solutions of nonlinear two point boundary value problems.

Qiao and $\mathrm{Li}$ [11] analyzed two kinds of important numerical methods forcalculating periodic solutions. TrungHieu [12] studied remarks on the shooting method for nonlinear two-point boundary value problem. Russell and Shampine [13] discussed numerical methods for singular boundary value problem. Sharma et al [14] studied numerical solution of two point boundary value problems using Galerkin-Finite element method. Hence the main objective of the present study is to solve nonlinear two point boundary value problems (BVP) by using simple and efficient shooting method. This well-known technique is an iterative algorithm which attempts to identify appropriate initial conditions for a related initial value problem that provides the solution to the original boundary value problem. 


\section{Mathematical Formulation}

For a general boundary value problem for a second-order ordinary differential equation, the simple shooting method is stated as follows:

$$
\begin{array}{r}
\text { Let, } x^{\prime \prime}(t)=f\left(t, x(t), x^{\prime}(t)\right), \quad t \in[a, b] \\
x(a)=\alpha, x(b)=\beta
\end{array}
$$

be the BVP in question and let $\mathrm{x}(\mathrm{t}, \mathrm{s})$ denote the solution of the IVP

$$
x^{\prime \prime}(t)=f\left(t, x(t), x^{\prime}(t)\right), t \in[a, b] \quad x(a)=\alpha, x^{\prime}(a)=s
$$

where $s$ is a parameter that can be varied. The IVP (2.2) is solved with different values of $\mathrm{s}$ with, e.g., Rung Kutta-4 method until the boundary condition on the right side $x(b)=\beta$ becomes fulfilled. As mentioned above, the solution $x(t, s)$ of (2.2) depends on the parameters. Let us define a function

$$
F(s):=x(b, s)-\beta
$$

If the BVP (2.1) has a solution, then the function $\mathrm{F}(\mathrm{s})$ has a root, which is just the value of the slope $x^{\prime}(a)$ giving the solution $\mathrm{x}(\mathrm{t})$ of the BVP in question. The zeros of $\mathrm{F}(\mathrm{s})$ can be found with, e.g., Newton's method.

Newton's method is probably the best known method for finding numerical approximations to the zeroes of a real-valued function. The idea of the method is to use the first few terms of the Taylor series of a function $F(s)$ in the vicinity of a suspected root, i.e.,

$$
F\left(s_{n}+h\right)=F\left(s_{n}\right)+F^{\prime}\left(s_{n}\right) h+Q\left(h^{2}\right)
$$

Where $s_{n}$ is the $n$ thapproximation of the root. Now if one inserts $h=s-s_{n}$, one obtains

$$
F(s)=F\left(s_{n}\right)+F^{\prime}\left(s_{n}\right)\left(s-s_{n}\right)
$$

As the next approximation $S_{n+1}$ to the root we choose the zero of this function, i.e,

$$
\begin{aligned}
& F\left(s_{n+1}\right)=F\left(s_{n}\right)+F^{\prime}\left(s_{n}\right)\left(s_{n+1}-s_{n}\right)=0 \\
& \Rightarrow s_{n+1}=s_{n}-\frac{F\left(s_{n}\right)}{F^{\prime}\left(s_{n}\right)}
\end{aligned}
$$

The derivative $F^{\prime}\left(s_{n}\right)$ can be calculated using the forward difference formula

$$
F^{\prime}\left(s_{n}\right)=\frac{F\left(s_{n}+\delta s\right)-F\left(s_{n}\right)}{\delta s}
$$

where $\delta s$ is small. Notice that this procedure can be unstable near a horizontal asymptote.

\section{Method of Solution Technique}

Consider the boundary value problem for the second-order differential equation of the form

$$
\begin{array}{r}
y^{\prime \prime}=p(x) y^{\prime}+q(x) y+r(x), \\
a \leq x \leq b, y(a)=\alpha, y(b)=\beta
\end{array}
$$

Then the two initial value problems is given by

$$
\begin{gathered}
y^{\prime \prime}=p(x) y^{\prime}+q(x) y+r(x), \\
a \leq x \leq b, y(a)=\alpha, y^{\prime}(a)=0 \\
y^{\prime \prime}=p(x) y^{\prime}+q(x) y+r(x), \\
a \leq x \leq b, y(a)=0, y^{\prime}(a)=1
\end{gathered}
$$

Then if $y_{1}(x)$ is the solution to (3.2) and $y_{2}(x)$ is the solution to equation (3.3) the solution to equation (3.1) is

$$
y(x)=y_{1}(x)+\frac{\beta-y_{1}(b)}{y_{2}(b)} y_{2}(x), y_{2}(b) \neq 0
$$

For the nonlinear case, the technique remains the same as that used to obtain a solution to equation (3.1) except that a sequence of initial value problems of the form;

$$
y^{\prime \prime}=f\left(x, y, y^{\prime}\right), a \leq x \leq b, y(a)=\alpha, y^{\prime}(a)=t_{k}
$$

where $t_{k}$ are real number are now required.

Let $y\left(x, t_{k}\right)$ be solution of the initial value problem(3.5).

We want to have a sequence $\left\{s_{k}\right\}$ so that

$$
\lim _{k \rightarrow \infty} y\left(x, t_{k}\right)=y(x)
$$

One of the choices for $s_{0}$ is;

$$
s_{0}=y^{\prime}(a)=\frac{y(b)-y(a)}{b-a}=\frac{\beta-\alpha}{b-a}
$$

Choosing the parameter $t_{k}$ for $k \geq 1$ to satisfy (3.6) is not easy and can be complicated by the fact that;

$$
y\left(b, t_{k}\right)-\beta=0
$$

is a nonlinear equation;

$$
\begin{gathered}
y^{\prime \prime}=f\left(x, y(x, t), y^{\prime}(x, t)\right), a \leq x \leq b, y(a, t)=\alpha, \\
y^{\prime}(a, t)=t_{k}
\end{gathered}
$$

The subscript $k$ is dropped inside the functional notation for convenience differentiating equation (3.7) with respect to $\mathrm{t}$ and assuming that the order of differentiation of $\mathrm{x}$ and $\mathrm{t}$ is reversible gives

$$
\begin{gathered}
\frac{\partial}{\partial t} y^{\prime \prime}(x, t)=\frac{\partial}{\partial y} f\left(x, y(x, t), y^{\prime}(x, t)\right) \frac{\partial}{\partial t} y(x, t)+ \\
\frac{\partial}{\partial y^{\prime}} f\left(x, y(x, t), y^{\prime}(x, t)\right) \frac{\partial}{\partial t} y^{\prime}(x, t) \\
\frac{\partial}{\partial t} y(a, t)=0, \quad \frac{\partial}{\partial t} y^{\prime}(a, t)=1
\end{gathered}
$$

Simplification using $z(x, t)$ to represent $\frac{\partial}{\partial t} y(x, t)$ results in 


$$
\begin{gathered}
z^{\prime \prime}=\frac{\partial}{\partial y} f\left(x, y, y^{\prime}\right) z+\frac{\partial}{\partial y^{\prime}} f\left(x, y, y^{\prime}\right) z^{\prime}, a \leq x \leq b, z(a)= \\
0, z^{\prime}(a)=1 \\
=f_{y} z+f_{y^{\prime}} z^{\prime}, a \leq x \leq b, z(a)=0, z^{\prime}(a)=1
\end{gathered}
$$

Finally, the Secant formula in general form is as follows:

$$
t_{k}=t_{k-1}-\frac{g\left(t_{k-1}\right)\left(t_{k-1}-t_{k}\right)}{g\left(t_{k-1}\right)-g\left(t_{k-2}\right)}
$$

We can update $t_{k}$ using the information from $z(x, t)$ as follows:

$$
t_{k}=t_{k-1}-\frac{\left(y\left(b, t_{k-1}\right)-\beta\right)}{z\left(b, t_{k-1}\right)}
$$

For a given accuracy $\epsilon$, the algorithm terminated if;

$$
\left.\mid y\left(b, t_{k-1}\right)-\beta\right) \mid<\epsilon .
$$

\section{Results and Discussion}

In this section, we explain five numerical examples of BVP which are available in the literature. The computations programming language, associated with the examples, are performed by MATLAB [15, 16, 17].

Example 1. Consider the boundary value problem;

$$
\begin{gathered}
y^{\prime \prime}=\frac{1}{8}\left(32+2 x^{3}-y y^{\prime}\right), 1 \leq x \leq 3, \\
y(1)=17, y(3)=\frac{43}{3}
\end{gathered}
$$

Solution: Let $f\left(x, y, y^{\prime}\right)=\frac{1}{8}\left(\left(32+2 x^{3}-y y^{\prime}\right)\right.$

$$
f_{y}=-\frac{1}{8} y^{\prime}, f_{y^{\prime}}=-\frac{1}{8} y
$$

Solve a system of two second- order initial value problems:

$$
\begin{gathered}
y^{\prime \prime}=\frac{1}{8}\left(32+2 x^{3}-y y^{\prime}\right), 1 \leq x \leq 3, y(1)=17, y^{\prime}(1)= \\
s_{k} \\
z^{\prime \prime}=f_{y} z+f_{y^{\prime}} z^{\prime}=-\frac{1}{8}\left(y^{\prime} z+y z^{\prime}\right), 1 \leq x \leq \leq 3, z(1)= \\
0, z^{\prime}(1)=1
\end{gathered}
$$

Let $u_{1}=y, u_{2}=y^{\prime}, u_{3}=z, u_{4}=z^{\prime}$.

Solve a system of four first-order initial value problems:

$$
\left\{\begin{array}{c}
u_{1}^{\prime}=u_{2} \\
u_{2}^{\prime}=\frac{1}{8}\left(32+2 x^{3}-u_{1} u_{2}\right) \\
u_{3}^{\prime}=u_{4} \\
u_{4}^{\prime}=-\frac{1}{8}\left(u_{2} u_{3}+u_{1} u_{4}\right)
\end{array} ; \quad\left[\begin{array}{l}
u_{1}(1)=17 \\
u_{2}(1)=s_{k} \\
u_{3}(1)=0 \\
u_{4}(1)=1
\end{array}\right]\right.
$$

Using MATLAB function ode45.m

Example 2.Consider the boundary value problem

$$
y^{\prime \prime}=-\left(y^{\prime}\right)^{2}-y+\ln (x), 1 \leq x \leq 2, y(1)=0, y(2)=
$$

Solution: Let

$$
\begin{gathered}
f\left(x, y, y^{\prime}\right)=-\left(y^{\prime}\right)^{2}-y+\ln (x), \\
f_{y}=-1, f_{y^{\prime}}=-2 y^{\prime}
\end{gathered}
$$

Solve a system of two second-order initial value problems:

$$
\begin{gathered}
y^{\prime \prime}=-\left(y^{\prime}\right)^{2}-y+\ln (x), 1 \leq x \leq 2, y(1)=0, y^{\prime}(1)=s_{k} \\
z^{\prime \prime}=f_{y^{z}} z+f_{y^{\prime}} z^{\prime}=-z-2 y^{\prime} z^{\prime}, 1 \leq x \leq 2, z(1)= \\
0, z^{\prime}(1)=1
\end{gathered}
$$

Let $u_{1}=y, u_{2}=y^{\prime}, u_{3}=z, u_{4}=z^{\prime}$.

Solve a system of four first-order initial value problems:

$$
\left\{\begin{array}{c}
u_{1}^{\prime}=u_{2} \\
u_{2}^{\prime}=-\left(u_{2}\right)^{2}-u_{1}+\ln (x) \\
u_{3}^{\prime}=u_{4} \\
u_{4}^{\prime}=-u_{3}-2 u_{2} u_{4}
\end{array} ;\left[\begin{array}{c}
u_{1}(1)=0 \\
u_{2}(1)=s_{k} \\
u_{3}(1)=0 \\
u_{4}(1)=1
\end{array}\right]\right.
$$

Using MATLAB function ode $45 . \mathrm{m}$

Example 3. Consider the boundary value problem

$$
y^{\prime \prime}=\frac{3}{2} y^{2}, 0 \leq x \leq 1, y(0)=4, y(1)=1
$$

Solution: Let $f\left(x, y, y^{\prime}\right)=\frac{3}{2} y^{2}$

$$
f_{y}=3 y, f_{y^{\prime}}=0
$$

Solve a system of two second-order initial value problem

$$
\begin{gathered}
y^{\prime \prime}=\frac{3}{2} y^{2}, 0 \leq x \leq 1, y(0)=4, y^{\prime}(0)=s_{k} \\
z^{\prime \prime}=f_{y} z+f_{y^{\prime}} z^{\prime}=3 y z, 0 \leq x \leq 1, z(0)=0, z^{\prime}(0)=1
\end{gathered}
$$

Let $u_{1}=y, u_{2}=y^{\prime}, u_{3}=z, u_{4}=z^{\prime}$.

Solve a system of four first-order initial value problem

$$
\left\{\begin{array}{c}
u_{1}^{\prime}=u_{2} \\
u_{2}^{\prime}=\frac{3}{2} u_{1}^{2} \\
u_{3}^{\prime}=u_{4} \\
u_{4}^{\prime}=3 u_{1} u_{3}
\end{array} ; \quad\left[\begin{array}{c}
u_{1}(0)=4 \\
u_{2}(0)=s_{k} \\
u_{3}(0)=0 \\
u_{4}(0)=1
\end{array}\right]\right.
$$

Using MATLAB function ode $45 . \mathrm{m}$

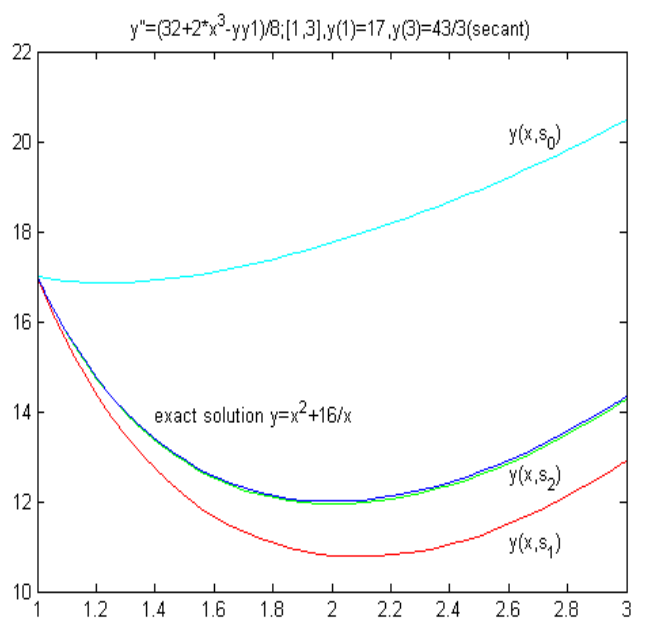

Figure 1. Plot of exact and approximate solution. 


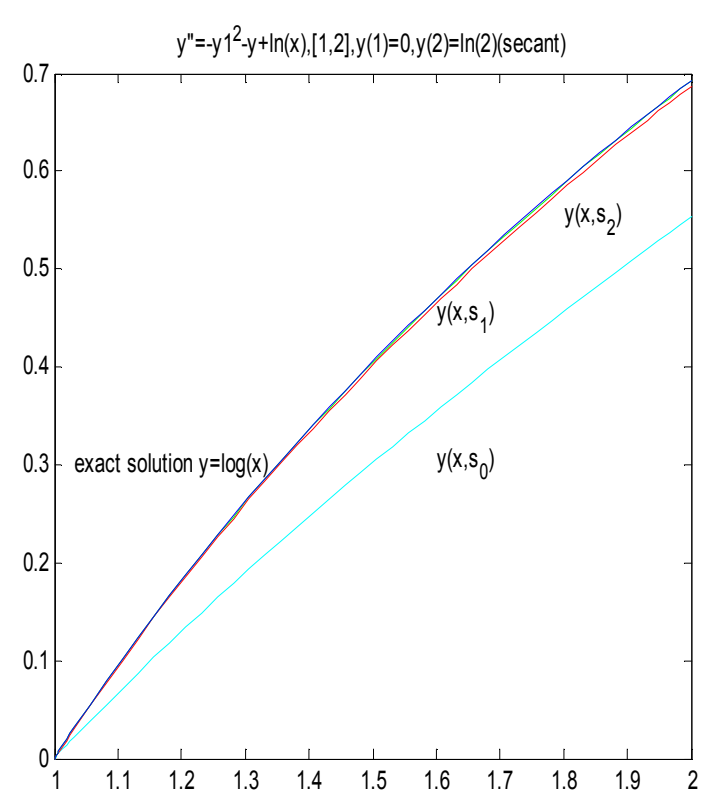

Figure 2. Plot of exact and approximate solution.

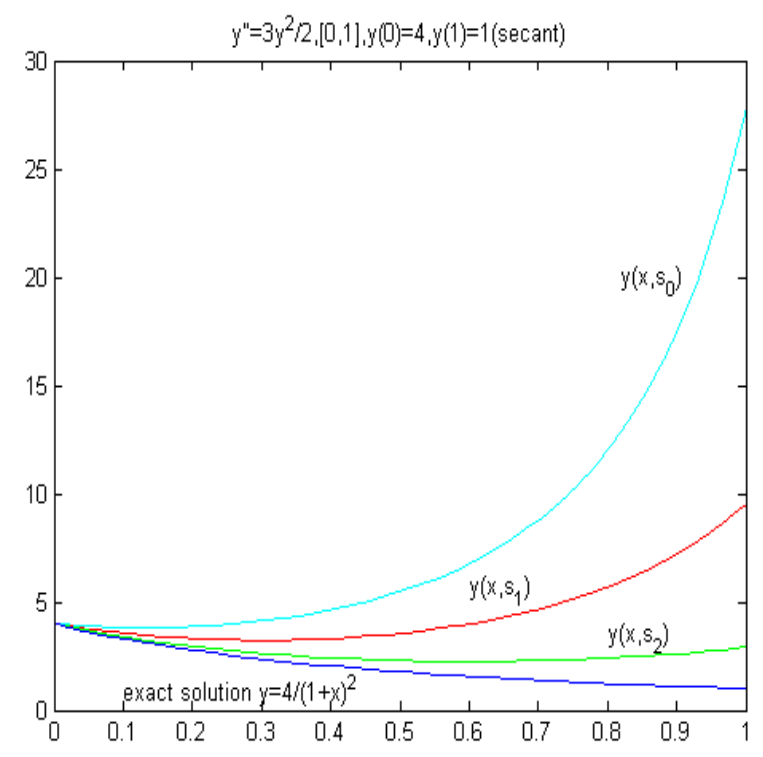

Figure 3. Plot of exact and approximate solution.

Figure 1, Figure 2 and Figure 3 shows that the approximate solution of Example 1, Example 2 and Example 3 is at the third iteration (shot), because the solution is almost coincident with the exact solution among the three shots.

Example 4.Consider the boundary value problem

$$
y^{\prime \prime}=y^{3}-y y^{\prime}, 1 \leq x \leq 2, y(1)=\frac{1}{2}, y(2)=\frac{1}{3}
$$

Solution: Let

$$
\begin{gathered}
f\left(x, y, y^{\prime}\right)=y^{3}-y y^{\prime} \\
f_{y}=3 y^{2}-y^{\prime}, f_{y^{\prime}}=-y
\end{gathered}
$$

Solve a system of two second-order initial value problems:

$$
y^{\prime \prime}=y^{3}-y y^{\prime}, 1 \leq x \leq 2, y(1)=\frac{1}{2}, y^{\prime}(1)=s_{k}
$$

$$
\begin{gathered}
z^{\prime \prime}=f_{y} z+f_{y^{\prime}} z^{\prime}=\left(3 y^{2}-y^{\prime}\right) z-y z^{\prime}, 1 \leq x \leq 2, z(1)= \\
0, z^{\prime}(1)=1
\end{gathered}
$$

Let $u_{1}=y, u_{2}=y^{\prime}, u_{3}=z, u_{4}=z^{\prime}$.

Solve a system of four first-order initial value problems:

$$
\left\{\begin{array}{c}
u_{1}^{\prime}=u_{2} \\
u_{2}^{\prime}=\left(u_{1}\right)^{3}-u_{1} u_{2} \\
u_{3}^{\prime}=u_{4} \\
u_{4}^{\prime}=3\left(u_{1}\right)^{2} u_{3}-u_{2} u_{3}-u_{1} u_{4}
\end{array} ;\left[\begin{array}{l}
u_{1}(1)=\frac{1}{2} \\
u_{2}(1)=s_{k} \\
u_{3}(1)=0 \\
u_{4}(1)=1
\end{array}\right]\right.
$$

Using MATLAB function ode45.m

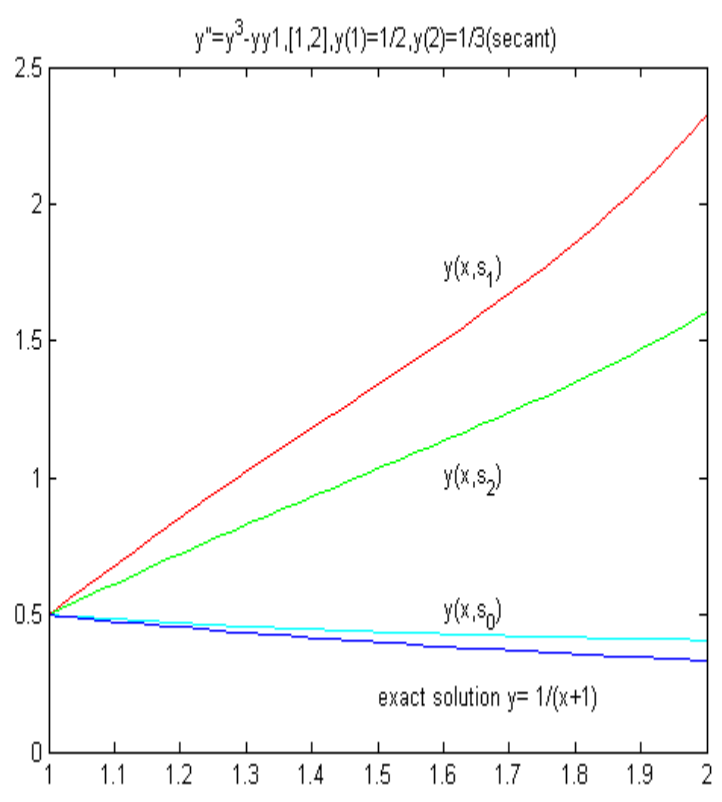

Figure 4. Plot of exact and approximate solution.

Example 5.Consider the boundary value problem

$$
\begin{gathered}
y^{\prime \prime}=y^{\prime}+2(y-\ln x)^{3}-\frac{1}{x}, 2 \leq x \leq 3, \\
y(2)=\frac{1}{2}+\ln 2, y(3)=\frac{1}{3}+\ln 3
\end{gathered}
$$

Solution: Let

$$
\begin{gathered}
f\left(x, y, y^{\prime}\right)=y^{\prime}+2(y-\ln x)^{3}-\frac{1}{x} \\
f_{y}=6(y-\ln x)^{2}, f_{y^{\prime}}=1
\end{gathered}
$$

Solve a system of two second-order initial value problems:

$$
\begin{aligned}
& y^{\prime \prime}=y^{\prime}+2(y-\ln x)^{3}-\frac{1}{x}, 2 \leq x \leq 3, y(2)=\frac{1}{2}+ \\
& \ln 2, y^{\prime}(2)=s_{k} \\
& z^{\prime \prime}=f_{y} z+f_{y^{\prime}} z^{\prime}=6(y-\ln x)^{2} z+z^{\prime}, 2 \leq x \leq 3, z(2)= \\
& 0, z^{\prime}(2)=1
\end{aligned}
$$

Let $u_{1}=y, u_{2}=y^{\prime}, u_{3}=z, u_{4}=z^{\prime}$.

Solve a system of four first-order initial value problems: 


$$
\left\{\begin{array}{c}
u_{1}^{\prime}=u_{2} \\
u_{2}^{\prime}=u_{2}+2\left(u_{1}-\ln (x)\right)^{3}-\frac{1}{x} ; \\
u_{3}^{\prime}=u_{4} \\
u_{4}^{\prime}=6 u_{3}\left(u_{1}-\ln (x)\right)^{2}+u_{4}
\end{array} \quad\left[\begin{array}{c}
u_{1}(2)=\frac{1}{2}+\ln (2) \\
u_{2}(2)=s_{k} \\
u_{3}(2)=0 \\
u_{4}(2)=1
\end{array}\right]\right.
$$

[3] Man KamKwong and James S. W. Wong, "The shooting method and non-homogeneous multipoint BVPs of second-order ODE,". Hindawi Publishing Corporation, Article ID 64012, 2007.

[4] W. M. Abd-Elhameed, E. H. Doha and Y. H. Youssri, "New wavelates collection method for solving second-order multipoint boundary value problems using Chebyshev polynomials of third and fourth kinds," Hindawi Publishing Corporation, Article ID 542839, 2013.

[5] Phang Pei See, Zanariah Abdul Majid and Mohamed Suleiman, "Solving nonlinear two point boundary value problem using two step direct method," Journal of Quality Measurement and Analysis, vol. 7, No. 1, pp. 129-140, 2011.

[6] Douglas B. Meade, Bala S. Haran and Ralph E. White, "The shooting technique for the solution two-point boundary value problems", 1996.

[7] M. M. Rahman, M.A. Hossen, M. Nurul Islamand Md. Shajib Ali, "Numerical Solutions of Second Order Boundary Value Problems by Galerkin Method with Hermite Polynomials," Annals of Pure and Applied Mathematics Vol. 1, No. 2, pp. 138-148, 2012.

[8] Afet Golayoglu Fatullayev, Emine Can and CananKoroglu, "Investigated numerical solution of a boundary value problem for a second order Fuzzy differential equation,". TWMS J. Pure Appl. Math, vol. 4, No. 2, pp. 169-176, 2013.

[9] A. Granas, R. B. Guenther and J. W. Lee, "The shooting method for the numerical solution of a class of nonlinear boundary value problems," SIAM J. Numer. Anal., vol. 16, No. 5, pp. 828-836, 2006.

Figure 4 and Figure 5 shows that the approximate solution of Example 4 and Example 5 is at the first iteration, because this solution is so close to the exact solution among the three shoots.

\section{Conclusion}

We have developed a Shooting method to solve non-linear two point boundary value problem analytically. The given problems were tested using three iterations of shooting method. In each figure, we represent the comparison between the exact solution and each iteration, which are made in order to solve these problems. The numerical results obtained by the proposed method are in good agreement with the exact solutions.

\section{References}

[1] D. J. Jones. "Use of a shooting method to compute Eigen-values of fourth-order two-point boundary value problems" Journal of Computational and Applied Mathematics, vol.47, pp. 395-400,1993.

[2] Huilan Wang, Zigen Ouyang and Liguang Wan, “Application of the shooting method to second - order multi- point integral boundary value problems," A Spinger open Journal ,Article ID 205,2013 .
[10] A. T. Cole and K. R. Adeboye, "Studied an alternative approach to solutions of nolinear two point boundary value problems," International Journal of Information and Communication Technology Research, vol. 3, No. 4, 2013.

[11] Tiantian Qiao and Weiguo Li, "Two kinds of important numerical methods forcalculating periodic solutions" Journal of Information and Communication science, vol. 1, pp. 85-92, 2006.

[12] Nguyen Trung Hieu, "Remarks on the shooting method for JOURNAL OF SCIENCE, Mathematics-Physics, 2003.

[13] R. D. Russell and L. F. Shampine, "Numerical methods for singular boundary value problem," SIAM. J. Numer. Anal., vol. 12, No. 1, pp. 13-36, 2006.

[14] Dinkar Sharma, Ram Jiuari and Sheo Kumar, "Numerical solution of two-point boundary value problems using Galerkin-Finite element method." International Journal of Nonlinear Science, vol. 13, No. 2, pp. 204-210,2012.

[15] L. F. Shampine, I. Gladwell and S. Thompson. Solving ODEs with MATLAB, 2003.

[16] L. F. Shampine, J. Kierzenka and M. W. Reichelt, "Solving boundary value problem for ordinary differential equations in MATLAB with bvp4c," 2000.

[17] Stephen J. Chapman, MATLAB Programming for Engineers, Thomson Learning, 2004. nonlinear two-point boundary value problem," VNU. 